\title{
Referee Acknowledgment
}

The Editors of Scientific Programming wish to acknowledge publicly the contributions of the following referees. The quality of a journal is influenced by the expertise and knowledge of its referees and we have been fortunate that the following have been willing to give of their knowledge and expertise to act as referees for Scientific Programming.

Adib Aliabadi, Queen's University, Belfast, $L K$ Peter Arbenz, ETH, Zürich, Switzerland

David Bailey, NASA Ames Research Center, Moffett Field, CA, LSA

Muhammad Benten, King Fahd L niversity of Petroleum \& Minerals, Saudi Arabia

Larry Berdahl, Lawrence Livermore National Laboratory, USA

Francine Berman. Lniversity of California, San Diego, USA

Robert Bjornson, Scientific Computing Associates, LSA

Hal Brand, Lawrence Livermore National Laboratory, USA

Ralph Brickner. Los Alamos National Laboratory, LSA

Mark Bull, Centre for Novel Computing, Lniversity of Manchester, $L K$

David Cann, Lawrence Livermore National Laboratory, USA

Glenn Carver, Cambridge Lniversity, $U K$

Barbara Chapman, Lniversity of Vienna, Austria

Margaret Chapman, Rogue Wave Software, LSA

Siddharta Chatterjee, NASA Ames Research Center, USA

Maurice Clint, Queen's Lniversity, Belfast, LK

John Conery, Lniversity of Oregon, USA

Philip Crooks. Queen's Lniversity, Belfast, $L K$

Lawrence Crowl. Oregon State Lniversity, LSA

Judy Cushing, Evergreen State College, LSA

Mike Delves, University of Liverpool, LK

Hank Dietz, Purdue Lniversity; USA

Bob Dobinson, CERN, Geneva, Switzerland

(C) 1995 by John Wiley \& Sons. Inc.

Scientific Programming. Vol. 4. pp. 119-121 (1995)

CCC $1058-9244 / 95 / 020119-03$
Paul Dubois, Lawrence Livermore National Laboratory, USA

Jeremy Du Croz. Numerical Algorithms Group, Oxford, $U K$

Iain Duff, Rutherford Appleton Laboratory, Did$\cot , U K$

Alan Edelmann, Department of Mathematics, MIT, Cambridge, MA, USA

Rudolf Eigenmann, University of Illinois, USA

Lars Eldén, Department of Mathematics, Linköping University, Sweden

Thomas Fahringer, University of Vienna, Austria

John Feo, Lawrence Livermore National Laboratory, USA

Alois Ferscha, University of Vienna, Austria

Rupert Ford, Centre for Novel Computing, Lniversity of Manchester. $U K$

Armando Fortuna, Centre for Novel Computing, University of Manchester, $L K$

Ian Foster, Argonne National Laboratory, LSA

Valery Fravssé. CERFACS, France

Len Freeman, University of Manchester, LK

Peter Fritzson, Department of Computing Science, Linköping University, Sweden

George Geist, Oak Ridge National Laboratory, Tennessee, USA

Wolfgang Gentzsch, Genias Software, Munich, Germany.

Michael Gerndt, KFA Jülich, Germany

Maya Gokhale, Supercomputing Research Center, USA

Brent Gorda, Lawrence Livermore National Laboratory, Germany

John Gurd, Centre for Novel Computing, University of Manchester, $U K$

Fred Gustavson, IBM T.J. Watson Research Center, Yorktown Heights, NY, USA

Matthew Haines, ICASE, USA

Dave Hardin, Lawrence Livermore National Laboratory, USA 
Terry Harmer, Queen's University, Belfast, UK

Ann Hayes, Los Alamos National Laboratory, USA

Rolf Hempel, GMD, St. Augustin, Germany

Tony Hey, University of Southampton, USA

Lee Higbie, Seki Systems, USA

Nick Higham, Department of Mathematics, University of Manchester, UK

Ladislav Hluchy, Slovak Academy of Sciences, Slovakia

Roger Hockney, University of Southampton, UK

Friedel Hossfeld, KFA Jülich, Germany

Robert Hotchkiss, Los Alamos National Laboratory, USA

Chris Jesshope, University of Surrey, $U K$

Barry Jones, University of Leicester, UK

Wouter Joosen, University of Leuven, Belgium

Charles Keolbel, Rice University, USA

Carl Kesselmann, California Institute of Technology, USA

Richard Kieburtz, Oregon Graduate iıistitute, USA

David Klappholz, Stevens Institute of Technology, USA

Adam Kolawa, Parasoft, USA

Ulrich Kremer, Rice University, USA

Anders Lansner, Department of Numerical Anal$y$ sis and Computing Science, Royal Institute of Technology (KTH), Stockholm, Sweden

David Levine, Argonne National Laboratory, Chicago, USA

Jen-Ki Li, Portland State University, LSA

Per Lindström, Department of Computing Science, Umeå University, Sweden

Per Ling, Department of Computing Science, Umeå University, Sweden

Per Lötstedt, Department of Scientific Computing, Uppsala University, Sweden

Olaf Lubeck, Los Alamos National Laboratory, USA

Robert Lyttle, Queen's University, Belfast, UK

John McCanny, Queen's Lniversity, Belfast, $L K$

Mike McCoy, Lawrence Livermore National Laboratory, USA

Tom MacDonald, Cray Research, Inc., LSA

Charles McDowell, University of California, Santa Cruz, USA

Jeanne Martin, Lawrence Livermore National Laboratory, USA

David Martinez, Sandia National Laboratories, USA

Eduard Mehofer, University of Vienna, Austria

Piyush Mehrotra, ICASE, USA

Patrick Miller, Lawrence Livermore National Laboratory, USA
Susan Mniszewski, Los Alamos National Laboratory, USA

Gary Montry, Southwest Software, USA

Peter Newton, University of Tennessee, USA

Andy Nisbet, Centre for Novel Computing, Lniversity of Manchester, $U K$

Dale Nielsen, Lawrence Livermore National Laboratory, USA

Sven Öberg, Department of Applied Mathematics, Luleå University of Technology, Sweden

Mathew O'Keefe, University of Minnesota, LSA

Steve Otto, Oregon Graduate Institute, USA

Ross Overbeek, Argonne National Laboratory, USA

David Padua, University of Illinois, USA

Cherri Pancake, Oregon State University, USA

Tom Patterson, Queen's University, Belfast, $U K$

Terry Pratt, University of Virginia, USA

Garth Reese, Sandia National Laboratories, USA

Graham Riley, Centre for Novel Computing, University of Manchester, $L K$

Karl Dieter Reinartz, University of Erlangen, Germany

Björn Roos, Department of Theoretical Chemistry, Lund University, Sweden

Dirk Roose, Department of Computer Science, Katholieke Universiteit, Leuven, Belgium

Matthew Rosing, Pacific Northwest Laboratory, USA

Axel Ruhe, Department of Computing Science. Chalmers University of Technology. Göteborg, Sweden

Joel Saltz, University of Maryland, LSA

Tom Scheffler, NASA Ames Research Center, USA

Robert Schreiber, NASA Ames Research Center, Moffett Field, CA, USA

Wolgang Schroeder-Preikschat, CMD First. Germany.

Daniel Segalman, Sandia National Laboratories, USA

Guiseppe Serazzi, Politecnico di Milano, Italy

Alex Shaferenko, University of Surrey, $L K$

Rob Sharp. Lawrence Livermore National Laboratory, USA

Margaret Simmons, Los Alamos National Laboratory, USA

Henk Sips, Technical Lniversity of Delft, Netherlands

David Skillicorn, Queen's University, Kingston, Canada

David Snelling, Centre for Novel Computing, Lniversity of Manchester, $L K$

Eric Stoltz, Oregon Graduate Institute, Oregon

Erik Tärnvik, Departmenı of Computing Science, Umeå University, Sweden 
Chau Wen Tseng, Rice University, USA

Michael Thuné, Department of Scientific Computing, Uppsala University, Sweden

Aimo Törn, Department of Computer Science, Ábo Academy University, NY, USA

Mustafa Uysal, University of Maryland, USA

Charles Van Loan, Department of Computer Science, Cornell University, NY, USA

Al Vermuelen, Rogue Wave Software, USA

Chris Wadsworth, Rutherford Appleton Laboratory, $U K$
Dick Wail, University of Liverpool, UK

Jonathan Walpole, Oregon Graduate Institute, USA

Ko Yang Wang, IBM, T. J. Watson Research Center, USA

Michael Wolfe, Oregon Graduate Institute, USA

Min-You Wu, SUNY Buffalo, USA

Mary Zosel, Lawrence Livermore National Laboratory, USA

Lin Zucconi, Lawrence National Laboratory, USA 

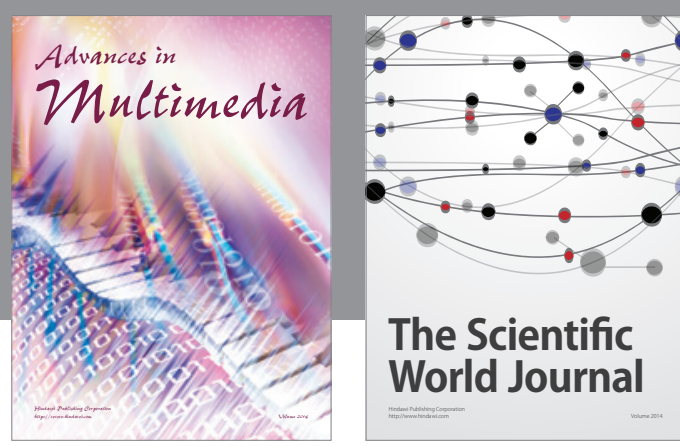

The Scientific World Journal
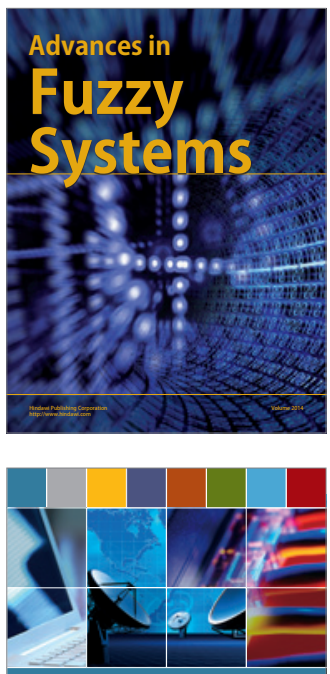

Computer Networks and Communications
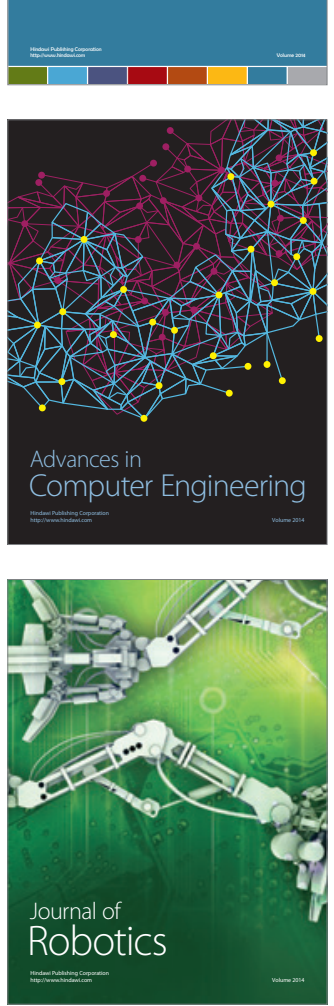
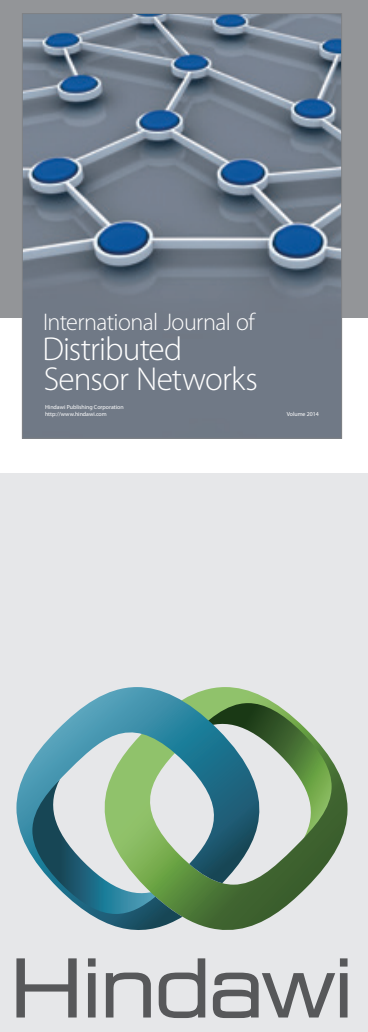

Submit your manuscripts at

http://www.hindawi.com
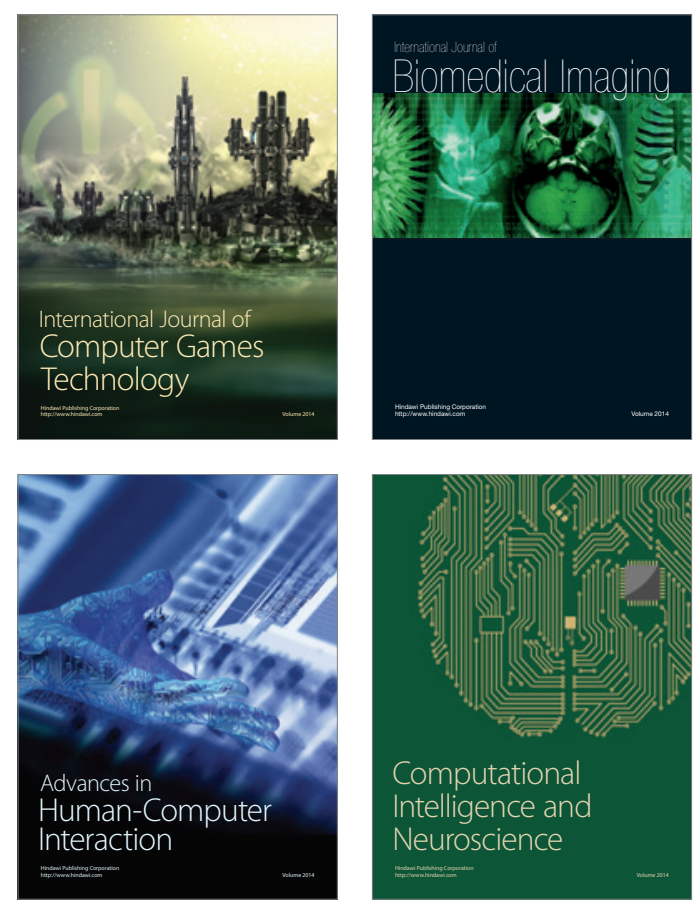
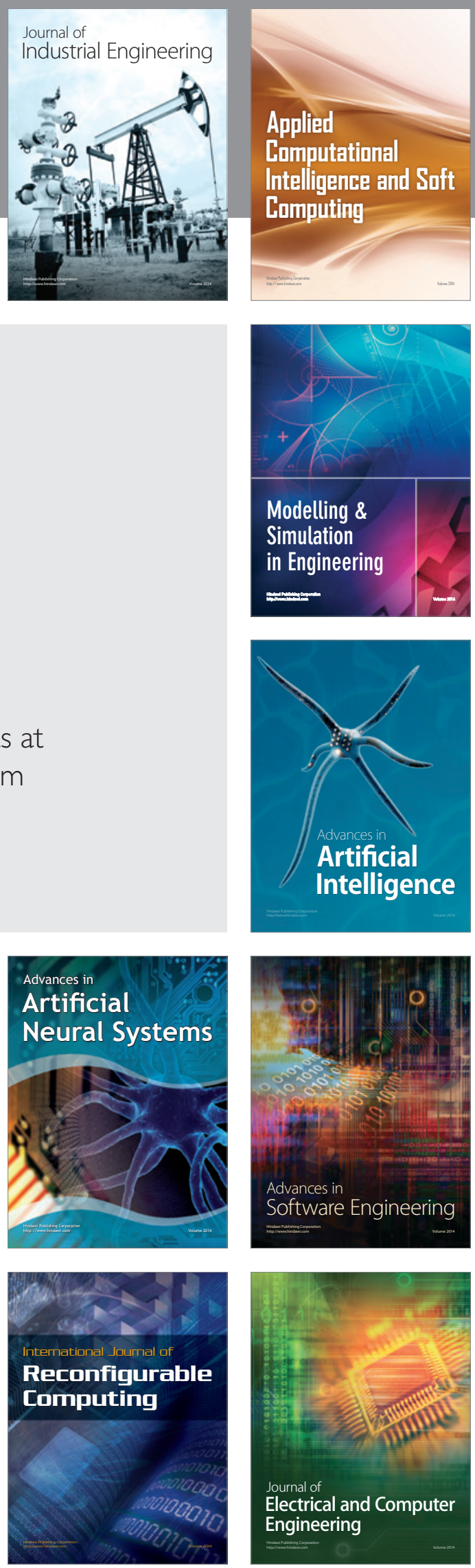